\title{
Comparison between Standard SMF and Non-zero Dispersion Shifted Fibre LEAF for Long Range Simultaneous Temperature and Strain Measurements
}

\author{
M. N. Alahbabi, Y. T. Cho and T. P. Newson \\ Optoelectronics Research Centre, University of Southampton \\ Southampton, SO17 1BJ, United Kingdom \\ Tel: +44 2380593836 Fax: +442380593149 \\ mna@orc.soton.ac.uk
}

\begin{abstract}
The relative accuracy of temperature and strain determination using Brillouin frequency shift and power change in standard single-mode fibre and the frequency shifts of the two Brillouin peaks in large effective area fibre is reported.

Keywords: Distributed Optical Sensors, Simultaneous Temperature and Strain Measurements, Spontaneous Brillouin Scattering.

\section{INTRODUCTION}

The dependence of the Brillouin frequency shift on strain and temperature has been previously reported as the basis for strain and temperature sensors but was unable to discriminate between the two measurands [1]. A development of this idea, is to use the Brillouin Optical Time-Domain Reflectometry (BOTDR) technique, in which both Brillouin frequency shift and change in power are used to obtain temperature and strain change simultaneously along a link of standard single mode fibre $(S M F)$; however range and measurand resolution are mainly limited by the power measurement accuracy. It was reported that $99.7 \%$ of the temperature error and $94 \%$ of the strain error is attributed to errors arising from the intensity measurement [2]. An elegant solution recently proposed by Lee et al. [3] is to use large effective area fibre $(L E A F)$, which has multiple composition fibre core. The Brillouin spectrum of silica fibre depends on it structure and doping composition of the fibre core. In $S M F$, only one peak is observed, whilst multiple peaks are observed in $L E A F$. The Brillouin frequency shifts of the first two peaks may be used to obtain the change in temperature and strain simultaneously, and avoids the need for a power measurement. This initial reported work was restricted to a relative short sensing fibre length of $3.7 \mathrm{~km}$. The novel aspect of this work is to explore whether this technique would prove advantageous for long range sensing, we have therefore compared experimentally the two methods.
\end{abstract}

\section{THEORY}

Lee et al. demonstrated that the frequency shift dependence of the Brillouin $1^{\text {st }}$ and $2^{\text {nd }}$ peaks are notably different for temperature, but similar for strain. Using the Brillouin frequency shift and power change matrix equation [4], but substituting the power change by the frequency shift of the $L E A F 2^{\text {nd }}$ peak, the Brillouin frequency shifts of peak one $\Delta V^{P 1}$ and peak two $\Delta V^{P 2}$ are related to temperature $T$ and strain $\varepsilon$ by the following relation:

$\Delta v^{P i}=C_{v T}^{P i} \Delta T+C_{v \varepsilon}^{P i} \Delta \varepsilon$

Where $\mathrm{i}=1,2$. Since the strain coefficients for $L E A F$ peaks $C_{v \varepsilon}^{P 1}$ and $C_{v \varepsilon}^{P 2}$ are equal, from (1), the change in temperature is given by (2). The change in fibre strain can be obtained by substituting the now known $\Delta T$ (2) into (1), and given in (3).

$$
\Delta T=\frac{\Delta v^{P 1}-\Delta v^{P 2}}{C_{v T}^{P 1}-C_{v T}^{P 2}}
$$

$$
\Delta \varepsilon=\frac{\Delta v^{P 2} C_{v T}^{P 1}-\Delta v^{P 1} C_{v T}^{P 2}}{C_{v T}^{P 1} C_{v \varepsilon}^{P 1}-C_{v T}^{P 2} C_{v \varepsilon}^{P 1}}
$$




\section{3-EXPERIMENTAL DETAILS}

The experimental arrangement is shown schematically in (Fig. 1).

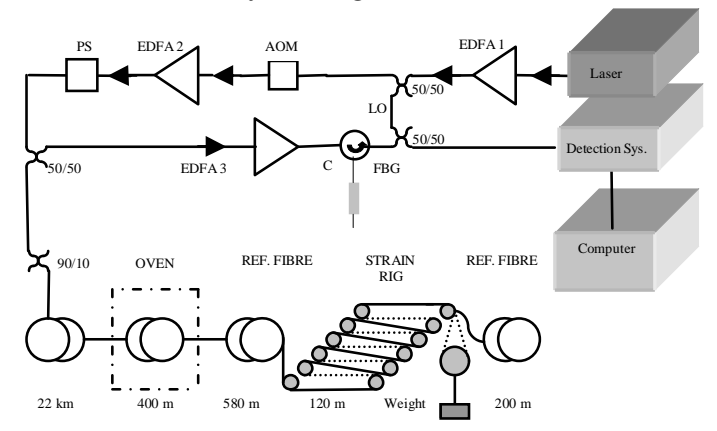

Figure 1: Schematic diagram of the experimental set-up.

The source consisted of a $100 \mu \mathrm{W}$ tuneable laser @ 1533.625nm and EDFA amplification, generating a probe pulse of $120 \mathrm{~mW}$, and 100ns duration. This was launched into the sensing fibre. Coherent detection of the spontaneous Brillouin backscatter signal was achieved by direct measurement of the $\sim 11 \mathrm{GHz}$ beat frequency between the optical local oscillator and the Brillouin signal using a wide bandwidth lightwave detector. The $L E A F$ investigated was Corning® Non-Zero Dispersion Shifted Single-Mode Fibre ( $L E A F ®)$ with the following characteristics: loss of $0.199 \mathrm{~dB} / \mathrm{km}$, effective area of $72 \mu \mathrm{m}^{2}$, and dispersion of $4.3 \mathrm{ps} / \mathrm{nm} . \mathrm{km} @ 1550 \mathrm{~nm}$. The SMF used in this comparison, had a loss of $0.2 \mathrm{~dB} / \mathrm{km}$ and an effective area of $\sim 80 \mu \mathrm{m}^{2}$. The sensing fibre was arranged as shown in figure 1 . The first $22 \mathrm{~km}$ remained on the original spools at room temperature. The next $400 \mathrm{~m}$ was loosely coiled and placed in an oven to allow heating whilst ensuring zero strain. The subsequent $580 \mathrm{~m}$ were used as a reference, maintained at room temperature with zero strain. The following $120 \mathrm{~m}$ section was passed round 6 pairs of pulleys, loaded by placing weights suspended at the end of the rig. The last $200 \mathrm{~m}$ was used as a second reference length and maintained at room temperature with zero strain. Initial tests were performed to establish valu es for the coefficients governing the frequency shifts and intensity variations with temperature and strain. The $L E A F$ was then heated and strained at the end of $23 \mathrm{~km}$. The Brillouin Anti-Stokes backscatter traces were collected for a number of frequencies over the heated and strained sections. The Brillouin spectrum at each point was obtained by fitting Lorentzian curves to the data. The Brillouin frequency shifts of each peak $P 1$ and $P 2$ and Brillouin power change were calculated and the temperature and strain computed using a) the two frequencies and b) the peak frequency and its intensity. The experiment was then repeated for $S M F$, measuring the frequency shift and power.

\section{RESULTS AND DISCUSSION}

The coefficients governing the frequency shifts and intensities variations with temperature and strain are shown in table 1. For the $S M F$ case, the measured coefficient values agreed closely with previously reported results [2, 5].

Table 1: LEAF and SMF Strain and Temperature Coefficients.

\begin{tabular}{llll}
\hline Coefficients & $\begin{array}{c}\text { LEAF } \\
\text { P1 }\end{array}$ & $\begin{array}{c}\text { LEAF } \\
\text { P2 }\end{array}$ & SMF \\
\hline$C_{v T}\left(\mathrm{MHz}^{\circ} \mathrm{C}^{-1}\right)$ & $1.0 \pm 0.086$ & $1.19 \pm 0.08$ & $1.07 \pm 0.070$ \\
$C_{v \varepsilon}\left(\mathrm{MHz} \mu \varepsilon^{-1}\right)$ & $0.051 \pm 0.003$ & $0.051 \pm 0.003$ & $0.048 \pm 0.003$ \\
$C_{P T}\left(\%{ }^{\circ} \mathrm{C}^{-1}\right)$ & $0.33 \pm 0.020$ & NA & $0.36 \pm 0.030$ \\
$C_{P \varepsilon}\left(\% \mu \varepsilon^{-1}\right)$ & $-7 \pm 6 \times 10^{-4}$ & NA & $-9 \pm 4 \times 10^{-4}$ \\
\hline
\end{tabular}




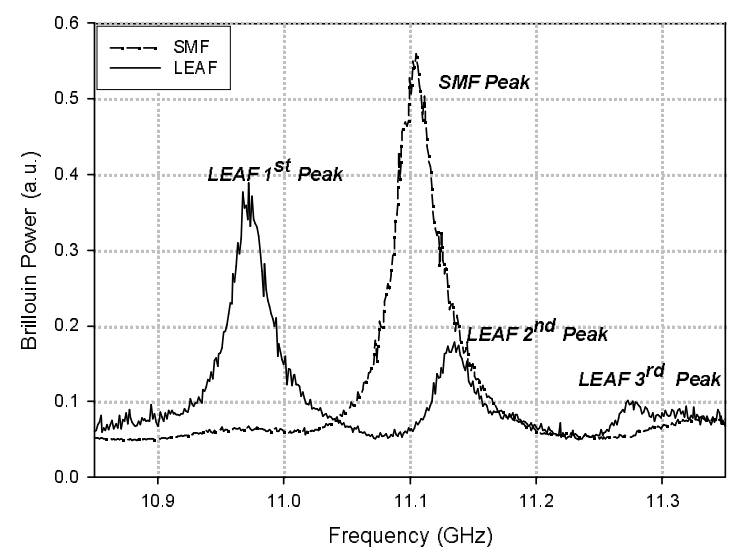

Figure 2: Brillouin spectrum in SMF and LEAF.

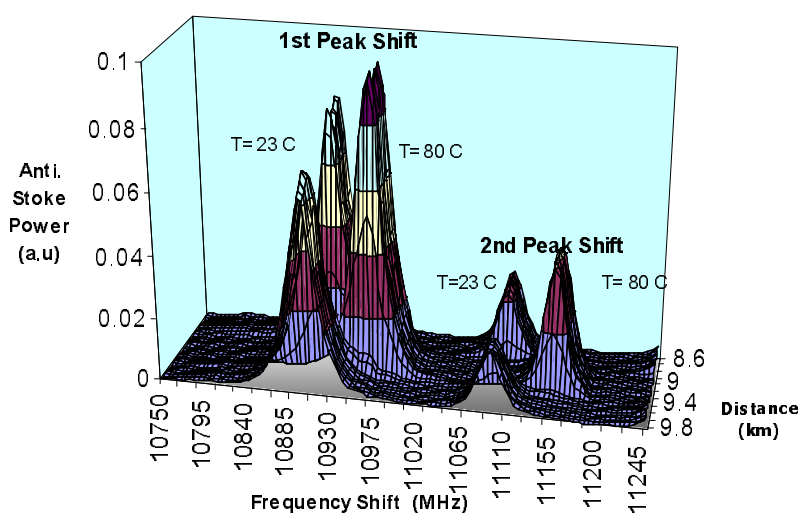

Figure 3: LEAF Brillouin frequency shifts at a heated section in LEAF.

As shown in figure 2, the Brillouin spectrum central frequencies at room temperature were $11.11 \mathrm{GHz}$ for $S M F, 10.91$ and $11.09 \mathrm{GHz}$ for $L E A F P 1$ and $P 2$ respectively. The total Brillouin $P 1$ and $P 2$ power was $43 \%$ of $S M F$ peak power. The line width of the Brillouin peaks in $L E A F \& S M F$ was measured to be approximately $35 \mathrm{MHz}$. For clarity, figure 3 shows a 3D plot of Brillouin frequency shift of $P 1$ and $P 2$ from $23^{\circ} \mathrm{C}$ to $80^{\circ} \mathrm{C}$ at a distance of $9 \mathrm{~km}$ along the sensing $L E A F$. Figures 4 and 5 shows the frequency shifts of $P 1$ and $P 2$ in the $L E A F$ and the frequency shift and power change in the $S M F$ respectively for the heated and strained sections over a range of temperatures, $23-80^{\circ} \mathrm{C}$, and strains, 0 $1600 \mu \varepsilon$.

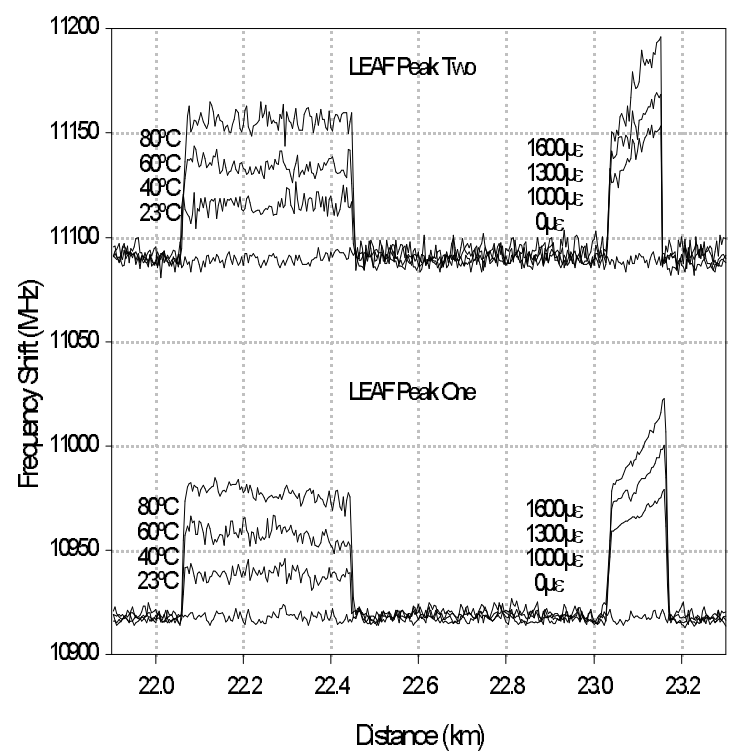

Figure 4: Brillouin frequency shifts at heated and strained sections in LEAF.

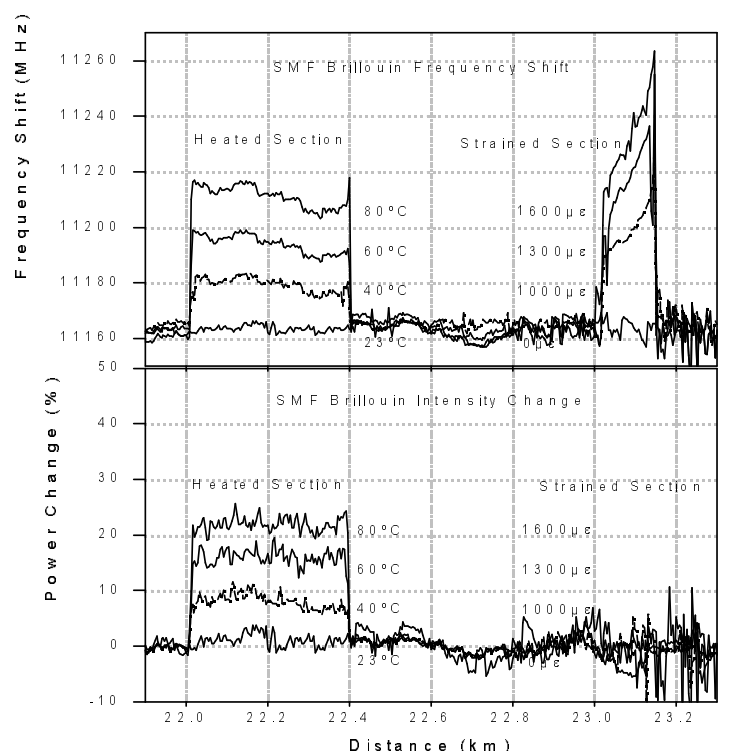

Figure 5: Brillouin frequency (top) and power change (bottom) at heated and strained sections in SMF.

The variation in frequency over the strain region indicates the friction in the pulley system. For further analysis, $L E A F$ $I^{s t}$ peak frequency shift and power change were used to obtain the change in temperature and strain simultaneousl $\mathrm{y}$, and the results are compared. For the heated and strained sections the frequency and power errors were calculated as shown in table 2 for the three cases: 
Table 2: Resolution of the frequency and power measurements in all three cases.

\begin{tabular}{|c|c|c|c|c|}
\hline Errors & $\begin{array}{l}\text { Sensing } \\
\text { Fibre }\end{array}$ & $\begin{array}{c}\text { LEAF } \\
\text { P1 }\end{array}$ & $\begin{array}{c}\text { LEAF } \\
\text { P2 }\end{array}$ & SMF \\
\hline \multirow[t]{2}{*}{ Frequency $(\mathrm{MHz})$} & Heated & 3.2 & 3.5 & 3.55 \\
\hline & Strained & 2.9 & 2.9 & 3.68 \\
\hline \multirow{2}{*}{$\begin{array}{l}\text { Power } \\
(\%)\end{array}$} & Heated & 3.5 & NA & 0.0146 \\
\hline & Strained & 4.5 & NA & 2.1200 \\
\hline
\end{tabular}

Using the values in table 2, the corresponding resolution of measured temperature and strain were calculated for all cases and listed in table 3 :

Table 3: Resolution of temperature and strain measurements in all three cases.

\begin{tabular}{lccc}
\hline \hline $\begin{array}{l}\text { Resolution } \\
\text { in three cases }\end{array}$ & LEAF & LEAF & SMF \\
\hline$\delta \mathrm{T}\left({ }^{\circ} \mathrm{C}\right)$ & 12 & 10 & 4.01 \\
$\delta \varepsilon(\mu \varepsilon)$ & 280 & 340 & 196 \\
\hline
\end{tabular}

As previously reported, in $S M F$, the resolution of the Brillouin power change measurement, limits the resolution of simultaneous temperature and strain measurements. Moreover; analysis of the LEAF $1^{s t}$ peak frequency shift and power change give higher temperature and strain errors due to it being a weaker signal. Analysis of the two frequency components of the $L E A F$ fibre, to determine the temperature and strain, produced a threefold increase in the temperature error from $4.01^{\circ} \mathrm{C}$ to $12^{\circ} \mathrm{C}$, and a $42 \%$ increase in the strain error compared to the $S M F$.

\section{CONCLUSION}

At present, the technique of using the two frequency peaks of the $L E A F$ to measure temperature and strain, yields increased errors as compared to the frequency and intensity analysis of the single frequency peak in $S M F$. Whilst our data collection system allows detection of the peak frequency to a resolution of less than $3.2 \mathrm{MHz}$ at this range, this new technique imposes more stringent requirements on the resolution of the frequency measurement; it is a differential frequency change that provides the temperature information i.e. $190 \mathrm{kHz} /{ }^{\circ} \mathrm{C}$ compared to $1 \mathrm{MHz} /{ }^{\circ} \mathrm{C}$. A temperature error of less than $1^{\circ} \mathrm{C}$ requires individual frequencies to be measured to a resolution of $\sim 100-150 \mathrm{KHz}$. The spontaneous Brillouin linewidth is approximately $35 \mathrm{MHz}$ and in the presence of noise will limit the resolution of the frequency measurement. Direct detection of the differential frequency may prove a means to achieve some improvement. It is concluded that distributed fibre sensors based on $L E A F$ or other fibres with multiple Brillouin peaks provide an exciting new possibility for extending their range and accuracy. However comparing the performance of dual frequency analysis of the Brillouin spectrum and peak frequency and intensity analysis of Brillouin spectra in $S M F$ we find a threefold increase in the temperature error and a $42 \%$ increase in strain error.

\section{AKNOWLEDGMENT}

The authors would like to thank Corning Optical Fibre for providing the LEAF fibre for this work.

\section{REFERENCES}

1. T. Kurashima, T. Horiguchi, and M. Teteda, Thermal effects of Brillouin gain spectra in single-mode fibers. IEEE Photonics Technology Letters, 1990. 2(10): p. 718-720.

2. S. M. Maughan, H. H. Kee, and T. P. Newson, Simultaneous distributed fibre temperature and strain sensor using microwave coherent detection of spontaneous Brillouin backscatter. Measurement Science \& Technology, 2001. 12(7): p. 834-842.

3. C. C. Lee, P. W. Chiang, and S. Shi, Utilization of a dispersion-shifted fiber for simultaneous measurement of distributed strain and temperature through Brillouin frequency shift. IEEE Photonics Technology Letters, 2001. 13(10): p. 1094-1096.

4. W. Jin, et al., Simultaneous Strain and Temperature Recovery: Error Analysis. Eleventh International Conference on Optical Fibre Sensors, 1966. Tu5(3): p. 116-119.

5. T. R. Parker, M.F., V. A. Handerek and A. J. Rogers, A fully distributed simultaneous strain and temperature sensor using spontaneous Brillouin backscatter. IEEE Photonics Technology Letters, 1997. 9(7): p. 979-981. 